\title{
Limb apraxia without aphasia from a left sided lesion in a right handed patient
}

\author{
Ola A Selnes, Alan Pestronk, John Hart, Barry Gordon
}

\begin{abstract}
A right handed man had a massive left middle cerebral artery stroke. CT and MRI revealed extensive destruction of both anterior and posterior areas typically associated with language. There was, however, no aphasia, but instead a marked limb apraxia, dyscalculia, dense right visual neglect, and anosognosia. These uncommon dissociations and associations support the hypothesis that cerebral control of motor function of the limbs is not fundamentally related to the motor control involved in speech, and the notion that handedness is related to laterality of motor control, and only accidentally to laterality of language control.
\end{abstract}

Aphasia and apraxia usually co-occur. ${ }^{1}$ A number of authors, ${ }^{23}$ have considered this association to be evidence that language and praxis share common mechanisms and common structures. It has also been suggested that the left hemisphere specialisation for language developed as a result of this hemisphere's pre-existing specialisation for certain motor activities that were also useful for purposes of communication. ${ }^{4}$ However, rare dissociations between language and praxis have been described, which suggest that the two are only coincidentally associated. ${ }^{5}$ In most of these cases, the dissociation has been between impairment of language and sparing of praxis. A confirmatory double dissociation, with impairment of praxis despite sparing of language, has rarely been seen. ${ }^{6}$ Even rarer has been the occurrence of apraxia without aphasia from a left-sided lesion in a right handed patient. ${ }^{7}$ We report another case of this latter dissociation. This case provides further evidence that language and limb praxis are not fundamentally related. The case is also of interest because it supports the hypothesis that handedness is more closely tied to the hemisphere dominant for motor skills than it is to the hemisphere dominant for language. ${ }^{8}$

\section{Case report}

An 81 year old right handed white male, with reasonably well controlled myasthenia gravis for 11 years, had sudden onset of right hemiparesis and inability to speak with intact speech comprehension. Neurological examination the following day showed a right homonymous hemianopsia with left gaze preference, a right hemiplegia involving the face, arm and leg, and a dense right hemisensory loss. Other changes included dysphagia, dysarthria, apraxia, right-sided neglect, dyscalculia, and finger agnosia. CT scan at that time was normal. A follow up scan two days later showed a very large left hemisphere infarction involving the frontal, parietal, and temporal lobes, including Broca's area, the insula, the posterior superior temporal gyrus and the supramarginal and angular gyrus, with associated mass effect, in the distribution of the middle cerebral artery (fig a). A subsequent MRI scan confirmed the infarction (fig b). There was no evidence by history, neurological examination or radiological studies to suggest an unrecognised right hemisphere lesion.

His hospital course was prolonged because of difficulties with swallowing not attributable to his myasthenia. During his 39 days in the hospital his neglect, anosognosia, apraxia, finger agnosia, and dyscalculia persisted unchanged; only a left gaze preference resolved.

He had neuropsychological evaluation 24, 25 and 32 days after onset, at which time his condition had stabilised. On formal testing, his attention/concentration abilities were mildly impaired, with a digit span forward of seven and a span backwards of three.

Assessment of language functions is summarised in table A. Spontaneous speech was dysarthric, hypophonic, and slowed, but fluent and meaningful without word finding difficulties or paraphasias. On the Cookie Theft picture, ${ }^{9}$ his responses were correct with cuing to the appropriate action in the picture. Verbal fluency $(F, A, S)$ was decreased, being partly attributable to his slow dysarthric output. Naming was mildly impaired with visual confrontation naming on the Boston Naming Test $^{10}$ initially $47 \%$ correct, but increasing to $66 \%$ correct on follow up testing. He performed at $80 \%$ correct on the BDAE body part naming subtest. Comprehension and repetition were intact. Oral reading and oral spelling were assessed by the Coltheart Reading List, ${ }^{11}$ which consists of 78 words with regular and irregular spellings. Performance was mildly impaired, with a score of $70 / 78$ correct. His errors were predominantly on the irregular words in both tasks. Reading comprehension of single words and the integrity of the semantic system at a word meaning level were normal as determined by a synonym judgement task. ${ }^{12}$ His general fund 

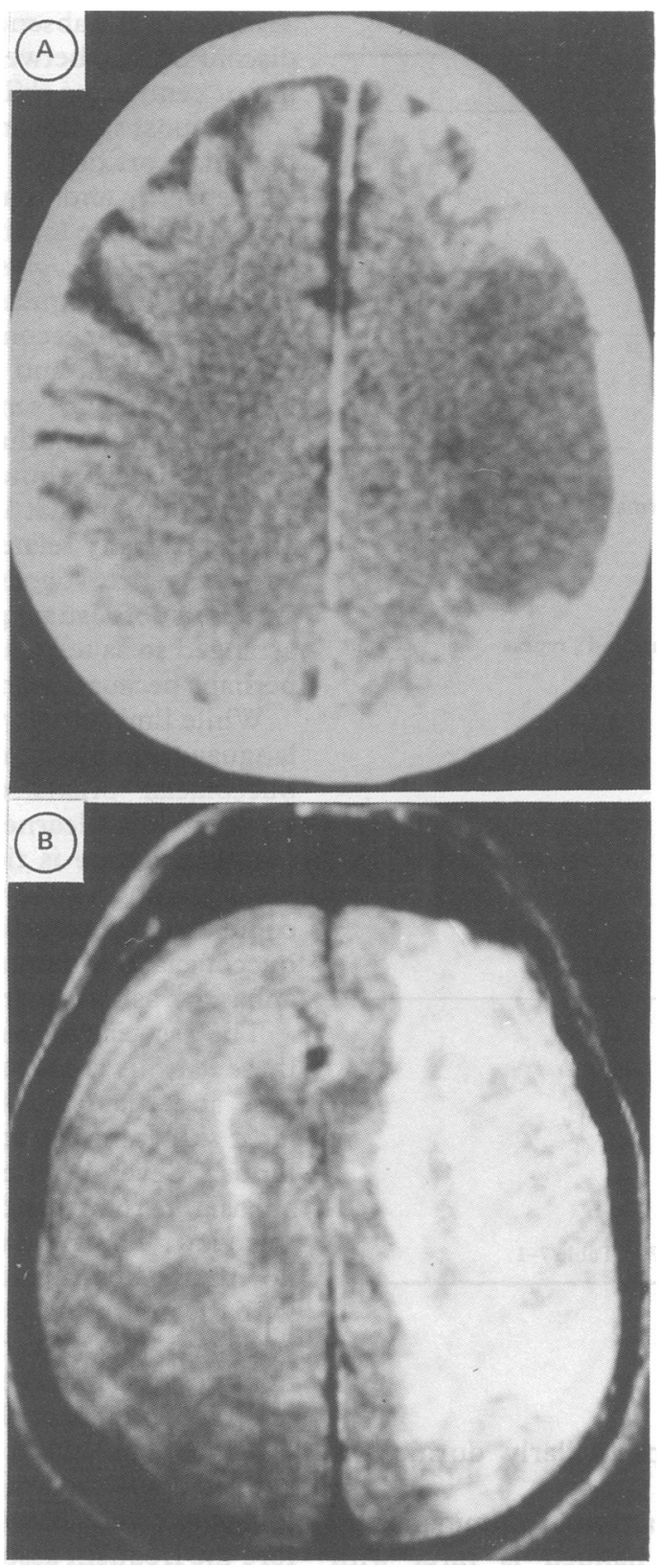

Figure a: Representative non-contrast $C T$ scans three days post-onset demonstrating the extent of the lefthemisphere infarction. In each section, right side of the brain is on the reader's left. $b$ : selections from $T 2$ weighted MRI images 21 days post onset demonstrating the extensive involvement of left-hemisphere language areas. In each section, right side of the brain is on the reader's left.

of verbal knowledge, as assessed by the information subtest of the WAIS-R, was normal (scaled score $=10$ ).

Written language skills could not be assessed due to his complete inability to write with his left hand secondary to the severity of his apraxia. Even with assistance in gripping the pen, he was unable to control the pen or form legible letters. Oral spelling, however, was within normal limits.

Testing for apraxia showed that buccofacial praxis was intact. On a test of Facial Praxis, ${ }^{13}$ he performed five out of six commands correctly, and recognised the correct gesture for his one error. On limb gesture on a praxis test, ${ }^{14}$ he performed $3 / 6$ on first presentation, and $4 / 6$ on second, with some improvement on demonstration of the gesture. On limb manipulation, however, he performed 0 out of 6 and 1 out of 6 with no improvement on demonstration of the gesture. When asked to demonstrate the use of a screwdriver, he positioned his hand as if holding a pen. When asked to show how to use a hammer, he maintained the same hand-position, but did move his hand up and down appropriately. A typical response for the serial motor act of folding a paper into thirds and placing it in an envelope was to fold the paper into sixteenths, hold the paper and envelope in one hand and wave them. Even with assistance to compensate for the hemiparesis and with demonstration, he continued to respond in this fashion. His performance on limb-commands deteriorated with increasing complexity of the command (table B).

Marked right sided neglect of body and room was noted. He was unable to point to body parts on his right side and did not recognise them as his own. He could point appropriately to objects on the left side of the room but not the right. He had no difficulties pointing to midline body-parts (for example, nose, mouth).

Visuo-constructional skills could not be fully assessed due to his inability to draw and copy. Right-left discrimination was intact. Finger identification was $3 / 5$ correct in identifying fingers to command. He could not point to fingers with his thumb, including those he could correctly identify. Oral calculations of addition and subtraction were within normal limits for simple addition/subtraction (13/18), but impaired for complex addition/ subtraction (0/5). He could not perform even simple multiplication or division orally.

He did not have insight into his illness despite being repeatedly informed of the nature of his problems. He apparently never ceased thinking he was hospitalised because of a broken leg, and persistently denied having had a stroke. When asked whether there was anything wrong with his right hand, he shook his left hand, and responded that it was fine.

He was transferred to a nursing home, where his dysarthria improved but his deficits otherwise remained stable. He had repeated bouts of pneumonia, and eventually died after several months.

\section{Discussion}

This patient did not develop an aphasia, despite CT and MRI evidence of extensive destruction of left hemisphere areas that would normally be involved in language processing. His language functions, including comprehension, repetition and expressive language, were normal, with the exception of mild-moderate deficits on visual confrontation naming. The relative sparing of linguistic functions implies that he was most likely an individual with reversed cerebral laterality for language. Even his mild naming deficits are consistent with this interpretation, since there is evidence that lesions of the non-dominant hemisphere may 
Table

A. Summary of results of language assessment

Spontaneous speech

Melodic line: 5-6 (mild impairment)

Phrase length: 4-5 (mildly reduced)

Articulatory agility: $3-4$ (mild-moderate impairment)

Grammatical form: 6-7 (normal)

Paraphasia: 7 (normal)

Word finding: $6-7$ (normal)

Auditory comprehension: 7 (normal)

Word Fluency (F, A, S):

4 words/min (impaired)

Auditory Comprehension:

Body part identification: $17 / 17$

Commands: $15 / 15$

Comprehension subtest of the WAIS-R: 10 (normal)

Auditory phoneme discrimination test (Wepman): $28 / 30$ (normal)

Repetition:

High probability sentences (BDAE)-8/8 (normal)

Naming:

Responsive naming (split half version BNT) 47\% (mildly impaired), repeat

$53 \%$ (mildly impaired), and repeat $66 \%$ (normal)

Body part naming: $8 / 10$ (mild impairment)

Oral Reading:

Coltheart Reading List 19/20 (normal); repeat testing: 70/76 (normal)

Reading regular words: $30 / 30$

Reading Comprehension:

Single word comprehension: $30 / 30$ (normal)

Oral Spelling:

Coltheart List: $18 / 20$ (normal)

B. Summary of results of praxis testing:*

Facial Praxis:

Buccofacial: 5/6 (normal)

Limb Apraxia:

Limb gesture: Trial 1: 3/6; Trial 2: 4/6

Demonstration: Trial 1: 3/3; Trial 2: $1 / 2$

Manipulation: Trial 1:0/6; Trial 2:1/6

Demonstration: Trial 1: 0/6; Trial 2: $1 / 5$

Serial complex acts: $0 / 2$

*Commands used are those given in Heilman and Valenstein (1979), Table 7-1.

result in dysnomia, particularly during the acute stage. $^{15}$

Right hemisphere language dominance in those that are right handed is rare, with estimates ranging from $1 \%{ }^{16}$ to $2 \cdot 6 \%{ }^{17}$ Only a handful of cases of aphasia with right-hemisphere lesions in right handed subjects have been reported at necropsy. ${ }^{18} \mathrm{Absence}$ of aphasia in a right handed subject, with an appropriate left hemisphere lesion, has been reported even less frequently. ${ }^{7}$ As was the case with our current patient, some of these reports note some subtle language deficits, but no obvious aphasic symptoms. This does not imply a less complete degree of language lateralisation in right handed subjects with right-hemisphere language dominance, since similar subtle problems of language processing have been noted in presumed left hemisphere dominant right handers with right hemisphere lesions. ${ }^{19}$

The fact that our patient was unable to write with his left hand, despite intact ability to spell orally, is consistent with previous descriptions of apractic agraphia. ${ }^{20}$ Previous cases of language sparing with left perisylvian lesions in right handed subjects have also reported disturbances of writing. The only two cases without any impairment of writing also did not have any limb or buccofacial apraxia, suggesting that agraphia in the absence of aphasia may reflect a disconnection between linguistic input and motor centres for skilled movements.

The most significant features of this case are that limb praxis was impaired while language was spared, and that handedness correlated with dominance for limb praxis rather than for language. The close association between apraxia, an acquired disorder of skilled movements not secondary to weakness or poor comprehension, and aphasia in most patients has suggested to some authors that the two share common mechanisms and neural structures. ${ }^{2}$ This case, however, provides additional documentation that language and limb praxis are not causally related. Rather, their frequent co-occurrence suggests that the neural substrates of both language and praxis are typically arranged so as to be affected by a single lesion, perhaps because of anatomical proximity. ${ }^{5}$

While limb praxis was clearly not related to language function in our patient, his spared buccofacial praxis might be construed as evidence that learned motor control of the mouth and face is tied to language function. This sparing in our patient may also be a coincidental association, since in other cases, buccofacial apraxia has been dissociable from aphasia, ${ }^{21}$ as well as from limb apraxia. ${ }^{22}$

The association that has been consistent in these various cases has been between handedness and the side of hemispheric control of most skilled motor activities of the hands and limbs. Several cases of so-called crossed aphasia in right handed subjects also support this view. The great majority of such cases do not report any apraxic difficulties, consistent with the interpretation that the left hemisphere remained the repository of skilled motor functions despite language functions being localised to the opposite hemisphere. Additional evidence comes from cases of those that are left handed who developed apraxia, but no aphasia, following right-hemisphere lesions. ${ }^{23}$ Therefore the frequent association between language dominance and handedness may also be an accident of anatomy, not from any fundamental interdependence between the two.

This work was supported in part by NIH-NINCDS TeacherInvestigator Award 1 K07 NS00721 to Barry Gordon. We thank Dr Michael McCloskey for helpful comments.

1 Kertesz A, Hooper P. Praxis and language: the extent and variety of apraxia in aphasia. Neuropsychologia 1981; 20:275-86.

2 Goldstein K. Language and language disturbances. New York: Grune and Stratton, 1948

3 Kimura D. Cerebral dominance for speech. In: D B Tower, ed. The nervous system. Vol 3: human communication and its ed. The nervous system. Vol 3: human comm

4 Kimura D, Archibald $Y$. Motor functions of the left hemisphere. Brain 1974;97:337-50.

5 Kertesz A, Ferro JM, Shewan CM. Apraxia and aphasia The functional-anatomical basis for their dissociation.
The Neurology 1984;34:40-47.

6 Heilman KM, Goneya EF, Geschwind N. Apraxia and agraphia in a right-hander. Cortex 1974;10:284-8.

7 Selnes OA, Risse GL, Rubens AB, Levy R. Transien aphasia with persistent apraxia. Uncommon sequela of massive left hemisphere stroke. Arch Neurol 1982;39: $122-6$

8 Brown JW, Wilson FR. Crossed aphasia in a dextral. Neurology 1973;23:907-11. 
9 Goodglass H, Kaplan E. The Assessment of aphasia and related disorders. Philadelphia: Lea and Febiger, 1972.

10 Goodglass H, Kaplan E, Weintraub S. The Revised Boston Naming Test. Philadelphia: Lea and Febiger, 1983

11 Coltheart M, Besner D, Jonasson JT, Davelaar E. Phonological encoding in the lexical decision task. $Q J$ Experi Psychol 1979;31:489-508.

12 Hart J, Gordon B. Delineation of single-word semantic comprehension deficits in aphasia with anatomical correlacomprehension deficits in aphasia
tion. Ann Neurol 1990;27:226-31.

13 Brown JW. Aphasia, apraxia and agnosia. Springfield, Ill: C C Thomas, 1972.

14 Heilman KM, Valenstein E. Clinical neuropsychology. New York: Oxford University Press, 1979.

15 Benson DF, Geschwind N. Aphasia and related disorders: A clinical approach. In: M-M Mesulam, ed. Principles of behavioural neurology. Philadelphia: F A Davis, 1985.

16 Gloning I, Gloning K, Haub G, Quatember R. Comparison of verbal behavior in right-handed an phere. Cortex 1969;5:43-52.

17 Roberts L. Aphasia, apraxia and agnosia in abnormal states of cerebral dominance. In: P J Vinken, $F$ W Bruyn, eds Handbook of clinical neurology, Vol 4. Amsterdam: Elsevier/North Holland, 1969.

18 April RS, Tse PC. Crossed aphasia in a Chinese bilingual dextral. Arch Neurol 1977;34:766-70.

19 Eisenson J. Language and intellectual modifications associated with right cerebral damage. Language and associated with righ
Speech 1962;5:49-53.

20 Valenstein E, Heilman KM. Apraxic agraphia with neglectinduced paragraphia. Arch Neurol 1979;36:506-8.

21 Kramer JH, Delis DC, Nakada T. Buccofacial apraxia without aphasia due to a right parietal lesion. Ann Neurol

22 Mani RB, Levine DN. Crossed buccofacial apraxia. Arch Neurol 1988;45:581-4.

23 Heilman KM, Coyle JM, Goneya EF, Geschwind N Apraxia and agraphia in a left-hander. Brain 1973;96: 21-28. 